\title{
Infeções do Trato Urinário numa Coorte de Transplantados Renais
}

\author{
Urinary Tract Infections in a Cohort of Kidney Transplant Recipients
}

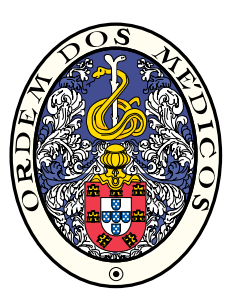

\author{
Ana BISPO ${ }^{1}$, Milene FERNANDES ${ }^{2}$, Cristina TOSCANO ${ }^{3}$, Teresa MARQUES ${ }^{3}$, Domingos MACHADO ${ }^{1}$, André WEIGERT $^{1}$ \\ Acta Med Port 2014 May-Jun;27(3):364-371
}

RESUMO

Introdução: A infeção do trato urinário é a complicação infeciosa mais comum no período pós transplante renal, estando a sua frequência pouco caracterizada na população portuguesa. Este trabalho teve como objetivo determinar a incidência de infeções do trato urinário e infeções do trato urinário recorrentes em transplantados renais.

Material e Métodos: Tratou-se de um estudo observacional de coorte retrospetiva, com consulta dos processos clínicos de doentes transplantados entre Janeiro de 2004 e Dezembro de 2005, no Hospital de Santa Cruz, com seguimento durante cinco anos ou até à data de perda de enxerto, morte ou perda de follow-up. Após uma análise descritiva da população, utilizámos testes bivariados para identificação de fatores associados a infeções do trato urinário.

Resultados: Em 127 doentes incluídos com seguimento de 593 doentes/ ano, 53 (41,7\%) tiveram pelo menos um episódio de infeção do trato urinário e $21(16,5 \%)$ tiveram infeções do trato urinário recorrentes. O género feminino foi o único fator associado com ocorrência de infeções do trato urinário $(p<0,001$, OR $=7,08, R R=2,95)$ e infeções do trato urinário recorrentes $(p<0,001, \mathrm{OR}=4,66$, $\mathrm{RR}=2,83)$. Os agentes etiológicos mais frequentes foram Escherichia coli $(51,6 \%)$, Klebsiella pneumoniae $(15,5 \%)$ e Enterobacter $\operatorname{spp}(9,9 \%)$. As infeções do trato urinário não causaram aumento de mortalidade ou perda de enxerto, mas foram a principal causa de internamentos hospitalares.

Discussão: Na nossa população, apenas o género feminino foi identificado como fator de risco para o desenvolvimento de infeções do trato urinário, recorrentes ou não. Escherichia coli foi o agente etiológico mais frequente.

Conclusão: Apesar das medidas preventivas adotadas, as infeções do trato urinário continuam a ser uma importante causa de morbilidade e de internamentos hospitalares.

Palavras-chave: Infeção do Tracto Urinário; Complicações Pós-operatórias; Factores de Risco; Transplantação Renal; Portugal.

\section{ABSTRACT}

Introduction: Urinary tract infection is the most common infectious complication following renal transplantation and its frequency is insufficiently studied in Portugal. The aim of this study was to characterize the incidence of urinary tract infections and recurrent urinary tract infections in renal transplant recipients.

Material and Methods: This was a retrospective cohort observational study, obtained from clinical files of all patients who received a renal transplant at the Hospital of Santa Cruz, from January 2004 to December 2005, with a mean follow-up period of five years or until date of graft loss, death or loss of follow-up. After a descriptive analysis of the population, we used bivariate tests to identify risk factors for urinary tract infections.

Results: A total of 127 patients were included, with a 593 patients.year follow-up. We detected 53 patients (41.7\%) presenting with at least one episode of urinary tract infection; 21 patients (16.5\%) had recurrent urinary tract infection. Female gender was the only risk factor associated with the occurrence of urinary tract infections $(p<0.001, \mathrm{OR}=7.08, \mathrm{RR}=2.95)$ and recurrent urinary tract infections $(p<0.001, \mathrm{OR}=4.66, \mathrm{RR}=2.83)$. Escherichia coli $(51.6 \%)$, Klebsiella pneumoniae $(15.5 \%)$ and Enterobacter spp $(9.9 \%)$ were the most frequently identified pathogens. Patients did not reveal an increased mortality or allograft loss. However, urinary tract infections were the most important cause of hospital admissions.

Discussion: Female gender was the only risk factor for urinary tract infections in this population. Escherichia coli was the most frequent agent isolated.

Conclusion: Despite preventive measures, urinary tract infections remain an important cause of morbidity and hospital admissions. Keywords: Urinary Tract Infections; Postoperative Complications; Risk Factors; Kidney Transplantation; Portugal.

\section{INTRODUÇÃo}

O transplante renal é, para muitos doentes, o método preferencial de substituição da função renal. ${ }^{1,2}$ Nos últimos anos, com a diminuição do risco de processos imunológicos conducentes à rejeição aguda do enxerto, aumentou a importância de outros mecanismos de lesão do enxerto, como a Infeção do Trato Urinário (ITU). ${ }^{2,3}$ A ITU é a infeção mais frequente no período pós transplante renal, variando a sua incidência entre 23 e $75 \%$, numa frequência supe- rior à observada na população em geral. ${ }^{1-6} \mathrm{~A}$ maioria das ITUs parece ocorrer no primeiro ano após transplante, com alguns estudos a indicar que as ITUs nos seis primeiros meses após transplante estão associadas a maior risco de perda de enxerto e maior mortalidade..$^{1-6}$ Uma complicação adicional são as ITUs recorrentes, que aumentam o risco de fibrose no enxerto com eventual deterioração funcional do mesmo. ${ }^{1,3,7}$ Pela sua recorrência, os microrganismos

1. Unidade de Transplantação Renal. Hospital de Santa Cruz. Centro Hospitalar de Lisboa Ocidental. Lisboa. Portugal.

2. Instituto de Medicina Preventiva. Faculdade de Medicina. Universidade de Lisboa. Lisboa. Portugal.

3. Laboratório de Microbiologia Clínica e Biologia Molecular. Hospital Egas Moniz. Centro Hospitalar de Lisboa Ocidental. Lisboa. Portugal.

Recebido: 02 de Novembro de 2013 - Aceite: 02 de Fevereiro de 2014 | Copyright @ Ordem dos Médicos 2014 
responsáveis podem desenvolver multirresistências, o que obriga ao uso de múltiplos esquemas de antibioterapia, nomeadamente de segunda linha e apenas disponíveis para administração endovenosa.

Estão descritos vários fatores de risco para a ocorrência de ITUs, recorrentes ou não, nos transplantados renais. ${ }^{1-3,5}$ Entre os fatores pré-transplante, estão incluídos o género feminino, a diabetes mellitus e alterações anatómicas do trato urinário (incluindo, nos homens, a hipertrofia prostática). ${ }^{1-3,5}$ Os procedimentos cirúrgicos na transplantação, principalmente aqueles relacionados com instrumentação do trato urinário, são também fatores associados às ITUs peri-transplante. ${ }^{1-3,5}$ Após transplante, destaca-se a imunossupressão como determinante major de ITUs. ${ }^{1-3}$

Apesar de existirem dados relativos à incidência de infeções na população de transplantados renais em Portugal, não se encontra discriminada qual a percentagem correspondente a ITUs. ${ }^{8}$ É, portanto, relevante verificar qual a sua frequência, que fatores estão implicados e quais os potencialmente modificáveis no contexto nacional. Numa perspetiva preventiva, interessa também caracterizar quais os microrganismos responsáveis pela maioria das ITUs pós-transplante, o que pode variar entre os diferentes centros nacionais. $^{2}$

O presente estudo teve como objetivo principal determinar qual a incidência de ITUs e ITUs recorrentes em indivíduos transplantados renais na Unidade de Transplantação Renal do Hospital de Santa Cruz - Centro Hospitalar de Lisboa Ocidental. Pretendemos ainda (1) avaliar a associação de ITUs e ITUs recorrentes com os fatores de risco descritos na bibliografia; (2) identificar diferenças na ocorrência de complicações no enxerto renal, nomeadamente, perda de enxerto por disfunção crónica e episódios de rejeição aguda, em indivíduos com ITUs e ITUs recorrentes; e (3) caracterizar as ITUs e ITUs recorrentes, quanto aos principais agentes etiológicos.

\section{MATERIAL E MÉTODOS}

Realizámos um estudo observacional de coorte retrospetiva, tendo como população alvo os doentes que receberam um transplante renal entre 1 de Janeiro de 2004 e 31 de Dezembro de 2005 no Hospital de Santa Cruz e que foram posteriormente seguidos na Consulta de Transplantação Renal do mesmo hospital. Para todos os doentes com estes critérios, consultámos os processos clínicos desde a data de transplante até cinco anos de seguimento ou até à data de perda de enxerto, morte ou perda de follow-up.

Definimos ITU quando existia registo no processo clínico de pelo menos um dos seguintes critérios: (1) urinocultura com mais de $10^{5}$ colónias/mL; (2) sinais clínicos de ITU, incluindo sintomas gerais (como mal-estar geral e febre com ou sem arrepios e/ou náuseas e vómitos) e/ou sintomas locais (como disúria, poliúria, urgência miccional, noctúria, desconforto suprapúbico, hematúria macroscópica e/ ou dor referida à região lombar ou ângulo costo-vertebral correspondente ou na fossa ilíaca ipsilateral ao enxerto renal), em indivíduos cujo hemograma revelasse leucocitose e/ou análise bioquímica com aumento da proteína $\mathrm{C}$ reativa e/ou exame sumário da urina característico (habitualmente com leucocitúria, podendo ter ainda $\mathrm{pH}$ ácido, piúria e/ou teste de nitritos positivos). Considerámos ITU recorrente quando ocorreram três ou mais episódios de ITU durante um período de 12 meses, ou dois episódios num período de seis meses.

Para cada doente analisámos as seguintes variáveis independentes e de caracterização geral: data de transplantação, idade nessa data, género, etiologia da insuficiência renal, tipo e duração da terapêutica de substituição renal (TSR) anterior ao transplante, tipo de dador (cadáver ou vivo), presença de diabetes mellitus à data de transplantação e o seu desenvolvimento durante o período de seguimento, esquema de imunossupressão de indução e de manutenção utilizados, esquema de profilaxia realizado, datas das infeções urinárias pós-transplante, microrganismos isolados em urinocultura, níveis de creatinina no seu nadir (12 meses pós-transplante) e no fim do seguimento. Registámos as causas e datas de perda de enxerto, bem como causas e datas de morte (quando aplicável).

Recolhemos os dados a partir dos processos clínicos dos doentes, para uma base de dados (Microsoft Excel ${ }^{\circledR}$ ) anonimizada e confidencial. Após uma primeira análise descritiva dos dados gerais da amostra, avaliámos a frequência de ITUs no período de seguimento. Para a análise de fatores associados às ITUs e ITUs recorrentes, considerámos o nível de significância de $5 \%$ para todos os testes - teste do qui-quadrado para as variáveis categóricas ou teste $t$ de student para as variáveis contínuas - utilizando o programa SPSS ${ }^{\circledR}$ para o Windows (versão 20.0; SPSS Inc., Chicago, IL, United States). Estimámos o risco relativo e respetivos intervalos de confiança (IC) a 95\% para as variáveis associadas com significado estatístico. Este projeto foi realizado após aprovação pela Comissão de Ética do CHLO.

\section{RESULTADOS}

\section{Caracterização sócio-demográfica e clínica dos doentes}

Estudámos uma coorte de 127 doentes, correspondendo a um total de 593 pessoas/ ano. Na Tabela 1, apresentamos os dados relativos à população total e aos casos de ITUs não recorrentes e recorrentes. A idade média ( \pm desvio-padrão) dos doentes à data de transplante era $43,0 \pm 15,0$ anos, sendo a coorte constituída por 58,3\% doentes do género masculino. À data de transplante, 9,4\% doentes tinham diabetes mellitus e 5,5\% desenvolveram-na após o transplante. A etiologia da doença renal crónica (DRC) mais comum foi a glomerulonefrite crónica $(26,8 \%)$, seguida da falência de transplante anterior $(11,8 \%)$ e vasculopatia (11,8\%). Previamente ao transplante, a TSR mais utilizada foi a hemodiálise $(77,2 \%)$ e a média de duração de TSR foi $41,9 \pm 36,8$ meses. Na maioria dos doentes $(77,2 \%)$ o enxerto renal teve origem em dador cadáver. O esquema de imunossupressão inicial mais utilizado foi a associação de ciclosporina $A$, micofenolato de mofetil e prednisolona $(78,0 \%)$, tendo-se registado o uso de terapêutica de in- 
Tabela 1 - Caracterização dos doentes na população total e comparando a) entre os que não desenvolveram e aqueles que tiveram pelo menos uma ITU e, deste último grupo $(n=53)$, comparando b) aqueles que registaram ITUs recorrentes com os que tiveram ITUs não recorrentes



Percentagens relativas à linha, exceto as assinaladas $\left({ }^{*}\right)$ calculadas para a população total - as percentagens apresentadas na comparação a) referem-se ao número de doentes com cada um dos fatores estudados na população total, enquanto as apresentadas na comparação b) referem-se ao número de doentes com cada um dos fatores estudados no grupo que teve pelo menos uma ITU. A negrito é indicada a única associação estatisticamente significativa, entre a variável sexo e a ocorrência de ITUs (valor $p<0,001$ ) e de ITUs recorrentes (valor $p<0,001$ ). dp, desvio-padrão. DRC, Doença Renal Crónica. TSR, Terapia de Substituição Renal. CsA, Ciclosporina A. MMF, Micofenolato de Mofetil. Pred, Prednisolona. Tac, Tacrolimus. ATG, globulina anti-timocítica. mTor, mammalian target of rapamycin.

dução de imunossupressão com globulina anti-timocítica (ATG), basiliximab ou ambos, em $46,5 \%$ dos doentes. $O$ uso de inibidores da mTor, em qualquer momento ao longo do seguimento, ocorreu em $31,5 \%$ da população. O nadir de creatinina sérica foi, em média, 1,42 $\pm 0,65 \mathrm{mg} / \mathrm{dL}$, sendo $1,69 \pm 1,41 \mathrm{mg} / \mathrm{dL}$ no fim do seguimento.

\section{Frequência das ITUs e ITUs recorrentes e avaliação de fatores associados}

Para análise das variáveis, dividimos a população em dois grupos principais: um grupo de doentes sem registo de ITUs ao longo do período de follow-up e um grupo de doentes com registo de pelo menos uma ITU. Este último grupo foi dividido em dois grupos subsequentes, comparando-se o grupo de doentes que não preenchiam os critérios para ITUs recorrentes com o grupo de doentes com ITUs recorrentes.

Ao longo do seguimento, $53(41,7 \%)$ doentes tiveram pelo menos uma ITU, correspondendo a uma taxa de incidência de 3,73 ITU por pessoa/ano. Considerando este grupo de doentes, $21(39,6 \%)$ registaram um ou mais casos de ITUs recorrentes (correspondendo a $16,5 \%$ da população total). O único fator de risco para o qual identificámos uma associação estatisticamente significativa com a ocorrência de ITUs e com a ocorrência de ITUs recorrentes foi o género (valor $p<0,001$ nas duas situações). As mulheres 
apresentaram uma probabilidade superior de desenvolver ITU, correspondendo a um risco relativo de 2,96 (IC95\%: 2,02-4,03) para a ocorrência de ITUs e de 2,83 (IC95\%: 0,96-8,32) para a ocorrência de ITUs recorrentes.

O tempo médio em TSR prévia ao transplante foi superior no grupo de doentes com ITUs (48,2 $\pm 37,0$ meses) relativamente ao grupo sem ITUs (37,5 \pm 36,2 meses). Registámos ITUs em $41,8 \%$ dos doentes que realizaram hemodiálise ( $n=98$ ) e em $36,8 \%$ dos que realizaram diálise peritoneal $(n=19)$ como TSR prévia ao transplante e, destes, $11,4 \%$ e $10,5 \%$ registaram ITUs recorrentes, respetivamente. Nos doentes em que foi utilizado o transplante preemptive (sem diálise prévia) $(n=2)$, não registámos ITUs.

As médias dos valores de creatininémia foram semeIhantes entre os dois grupos aos 12 meses $(1,43 \mathrm{mg} / \mathrm{dL}$ no grupo sem ITUs e $1,41 \mathrm{mg} / \mathrm{dL}$ no grupo em que ocorreu pelo menos uma ITU) notando-se, aos cinco anos, um valor superior de creatininémia, apesar de não significativo, no grupo com ITUs (1,65 mg/ dL no grupo sem ITUs e 1,75 $\mathrm{mg} / \mathrm{dL}$ no grupo em que ocorreu pelo menos uma ITU). No grupo de doentes com ITUs recorrentes registámos um valor de creatininémia inferior quer aos 12 meses, quer aos cinco anos $(1,25 \mathrm{mg} / \mathrm{dL}$ e $1,53 \mathrm{mg} / \mathrm{dL}$, respetivamente) relativamente ao grupo de doentes com ITUs não recorrentes. Contudo a diferença entre o valor de creatininémia no seu nadir e aos cinco anos foi semelhante $(0,37 \mathrm{mg} / \mathrm{dL}$ no grupo com ITUs não recorrentes e $0,28 \mathrm{mg} / \mathrm{dL}$ no grupo com ITUs recorrentes).

\section{Resultados clínicos na população total e nos grupos com ou sem ITUs}

Como indicamos na Tabela 2, 11,0\% dos doentes perderam o enxerto renal durante o seguimento, sendo a rejeição aguda a principal causa (35,7\%). Dos doentes em que a disfunção crónica constituiu a causa de perda do enxerto,
$75,0 \%$ registou pelo menos uma ITU ao longo do seguimento. Relativamente aos doentes que perderam o enxerto, a duração média deste foi $24,1 \pm 19,3$ meses. Durante os cinco anos de seguimento, apenas um doente faleceu (quatro anos após o transplante), não sendo conhecida a causa de morte e nunca se tendo registado ITUs neste doente. De referir que não houve perda em follow-up de nenhum dos doentes da coorte.

\section{Aspetos clínicos e etiológicos das ITUs recorrentes e não recorrentes}

Durante o primeiro semestre pós-transplante ocorreram $25,5 \%$ de todos os episódios de ITUs (Fig. 1). O tempo médio para ocorrência da primeira ITU pós transplante foi $13,06 \pm 14,43$ meses.

Houve necessidade de internamento por pielonefrite aguda em $30,6 \%$ dos episódios de ITUs não recorrentes e em $28,3 \%$ nos episódios de ITUs recorrentes. As ITUs foram a principal causa de internamento no Serviço de $\mathrm{Ne}$ frologia. (Fig. 2).

Considerando o total dos episódios de ITU (Fig. 3), o agente mais frequentemente isolado em urinocultura foi Escherichia coli $(51,6 \%)$, seguido de Klebsiella pneumoniae $(15,5 \%)$ e Enterobacter spp. (9,9\%). Registámos um número superior de ITUs causadas por Escherichia coli no grupo de ITUs não recorrentes $(59,7 \%)$ relativamente ao grupo de ITUs recorrentes (46,5\%), bem como por Proteus mirabilis (4,8\% vs 1,0\%). Observamos um maior número de ITUs por Enterobacter spp. (12,1\%), Enterococcus faecalis $(9,1 \%)$, Klebsiella pneumoniae $(18,2 \%)$ e Staphylococcus spp. $(4,0 \%)$ no grupo de doentes com ITUs recorrentes.

\section{DISCUSSÃO}

Entre os recetores de transplante renal no Hospital de Santa Cruz nos anos de 2004 e 2005, 41,7\% teve pelo menos um episódio de ITU durante os 5 anos de período de

Tabela 2 - Resultados clínicos dos doentes da população total e do grupo com pelo menos uma ITU ao longo do período de seguimento

\begin{tabular}{|c|c|c|c|c|c|}
\hline \multirow[b]{2}{*}{ Resultados Clínicos } & \multirow[b]{2}{*}{$\begin{array}{c}\text { Total } \\
(n=127)\end{array}$} & \multicolumn{2}{|r|}{ a) } & \multicolumn{2}{|c|}{ b) } \\
\hline & & $\begin{array}{l}\text { Grupo sem ITUs } \\
\qquad(n=74)\end{array}$ & $\begin{array}{l}\text { Grupo em que ocorreu } \\
\text { pelo menos uma ITU } \\
\qquad(n=53)\end{array}$ & $\begin{array}{l}\text { ITUs não } \\
\text { recorrentes } \\
(n=32)\end{array}$ & $\begin{array}{l}\text { ITUs recorrentes } \\
\quad(n=21)\end{array}$ \\
\hline Com enxerto funcionante, $n(\%)$ & $112(88,2)$ & $65(87,8)$ & $47(88,7)$ & $29(90,6)$ & $18(85,7)$ \\
\hline Perda de enxerto, $n(\%)$ & $14(11,0)$ & $8(10,8)$ & $6(11,3)$ & $3(9,4)$ & $3(14,3)$ \\
\hline \multicolumn{6}{|l|}{ Motivo de perda de enxerto, $n(\%)^{*}$} \\
\hline Rejeição aguda & $5(35,7)$ & $4(50,0)$ & $1(16,7)$ & $1(33,3)$ & $0(0,0)$ \\
\hline Disfunção crónica do enxerto & $4(28,6)$ & $1(12,5)$ & $3(50,0)$ & $1(33,3)$ & $2(66,7)$ \\
\hline Complicações vasculares & $2(14,3)$ & $2(25,0)$ & $0(0,0)$ & -- & -- \\
\hline Recidiva da doença base & $1(7,1)$ & $1(12,5)$ & $0(0,0)$ & -- & -- \\
\hline Outras / Indeterminado & $2(14,3)$ & $0(0,0)$ & $2(33,3)$ & $1(33,3)$ & $1(33,3)$ \\
\hline Morte, $n(\%)$ & $1(0,8)$ & $1(1,4)$ & $0(0,0)$ & -- & -- \\
\hline
\end{tabular}

Percentagens calculadas em coluna, em função do total de doentes para cada grupo, exceto onde assinalado $\left(^{*}\right)$ em que as percentagens foram calculadas em função dos doentes com perdas de enxerto, para cada grupo 


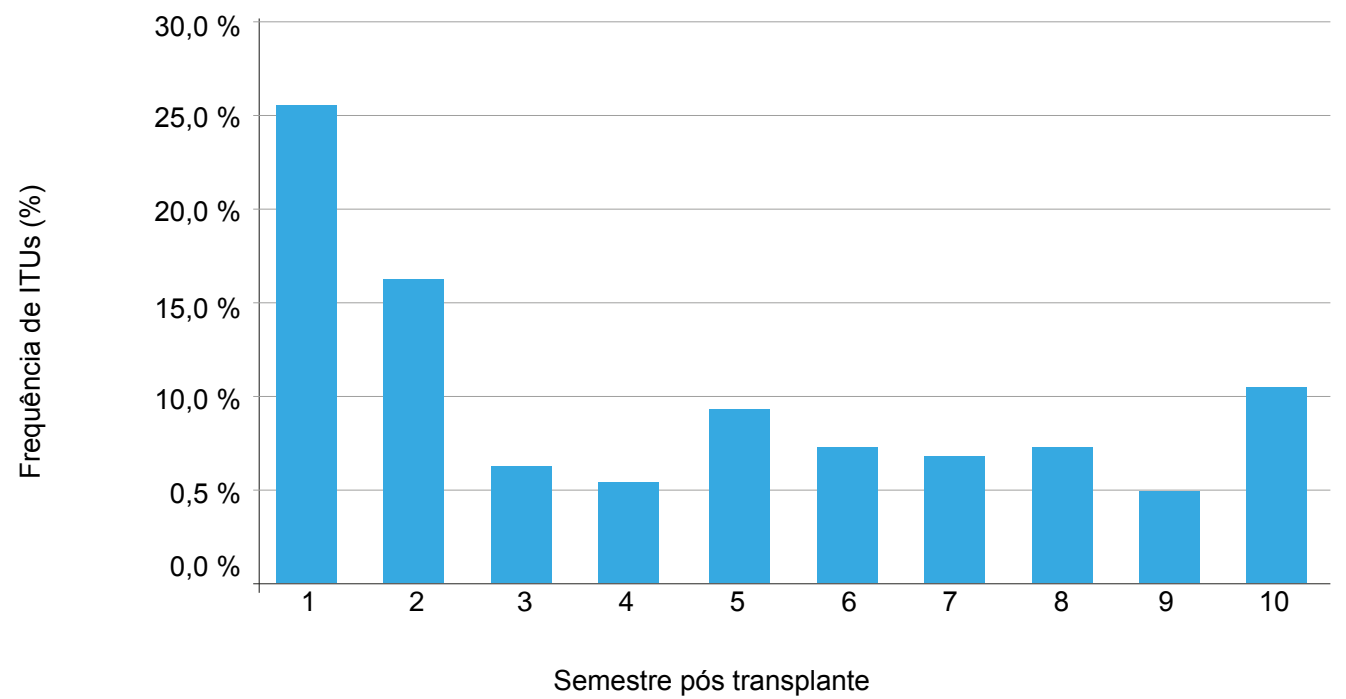

Figura 1 - Número de ITUs ao longo dos semestres de seguimento

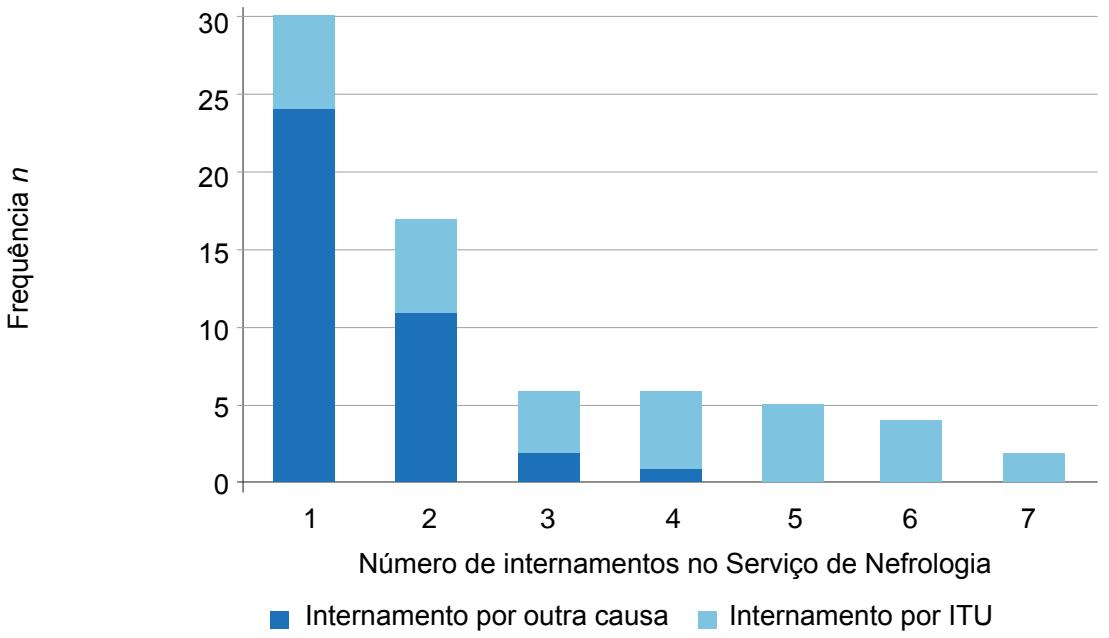

Figura 2 - Número de indivíduos de acordo com o número total de internamentos por doente no Serviço de Nefrologia do Hospital de Santa Cruz

seguimento, o que se enquadra nos valores descritos na literatura. ${ }^{1,2,6,9} \mathrm{~A}$ incidência de $16,5 \%$ referente às ITUs recorrentes é também semelhante ao referido na bibliografia. ${ }^{5,9}$ Um estudo realizado recentemente no Centro Hospitalar e Universitário de Coimbra apresentou uma incidência de ITUs recorrentes de $20,6 \%$ dos doentes. ${ }^{10}$

Existem vários fatores que têm sido associados a uma maior incidência de ITUs, nomeadamente fatores peri-operatórios e características clínicas e sociais do doente. .,2,5,7,11 $^{2}$ No entanto, apenas observámos como associação estatisticamente significativa a verificada entre o género feminino e a ocorrência de ITUs e de ITUs recorrentes. Esta é uma associação bem estabelecida nesta população, consistente em variados estudos realizados. ${ }^{1,2,6,11}$

Apesar da presença de DM e o seu desenvolvimento ao longo do período de seguimento estarem documentados como fatores de risco para ITUs ${ }^{1-3,9}$ à semelhança do que aconteceu noutros estudos, ${ }^{1,6,12}$ não foi possível estabelecer essa associação. Apenas $5,5 \%$ da população estudada desenvolveu DM durante o período de seguimento, um valor inferior ao relatado na literatura (atingindo valores de $13 \%$, cinco anos após transplante).${ }^{13}$ Apesar de algumas etiologias de DRC estarem associadas a uma maior ocorrência de ITUs, tais como a pielonefrite intersticial associada a RVU sem obstrução, a bexiga neurogénica e a uropatia obstrutiva, ${ }^{1-3,5,11}$ tal relação não foi apurada neste trabalho. A duração da TSR anterior ao transplante foi ligeiramente superior no grupo em que ocorreu pelo menos uma ITU não existindo, contudo, uma associação estatisticamente significativa. Sabe-se que um tempo mais prolongado em TSR pode levar a uma diminuição ou perda total de diurese residual, podendo associar-se a uma profunda alteração da anatomia das vias urinárias, com predisposição para a ocorrência de ITUs. ${ }^{5,9}$

Mostra-se interessante o facto de os doentes que realizaram transplante antecipado (preemptive) não terem registado nenhuma ITU $(n=2)$, o que pode ser reflexo de um grupo de doentes com um melhor estado clínico, uma vez que não sofreram as complicações que os tratamentos dialíticos condicionam tendo, necessariamente, uma 


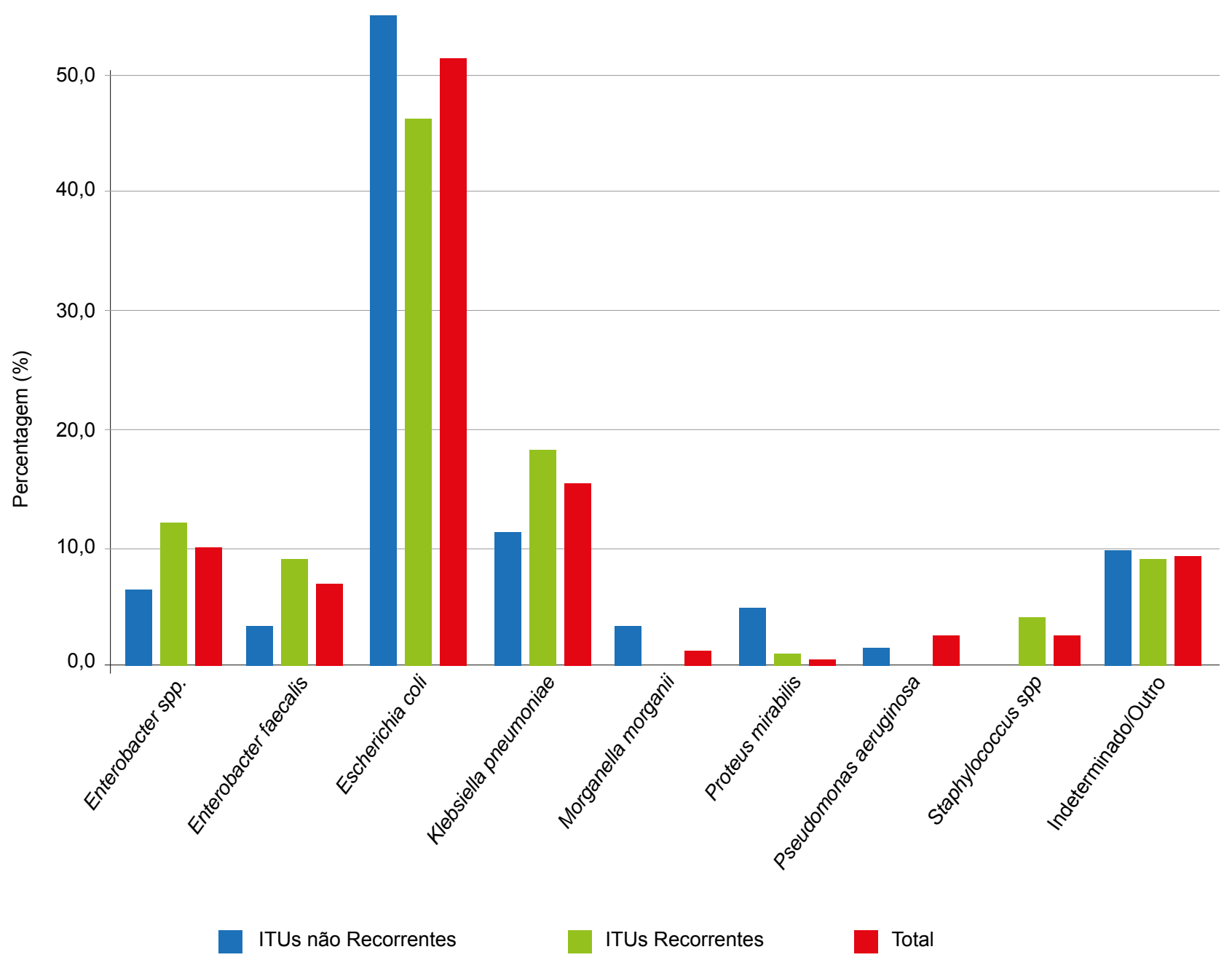

Figura 3 - Comparação entre os agentes etiológicos dos episódios de ITUs não recorrentes, ITUs recorrentes e o total de episódios de ITUs

excelente diurese pré-transplante. Não é possível, contudo, retirar conclusões desta tendência pelo reduzido número de casos em que foi utilizada esta modalidade terapêutica.

A maioria dos doentes tinha enxerto proveniente de cadáver $(77,2 \%)$, o que reflete também a realidade nacional ${ }^{8}$ estando documentada uma associação positiva entre a origem cadáver do enxerto e a ocorrência de ITUs, possivelmente devido ao enxerto estar sujeito a um período de isquemia mais prolongado e poder ser mais facilmente contaminado. ${ }^{1-4,6}$

Relativamente ao esquema de imunossupressão utilizado, não conseguimos estabelecer associações estatisticamente significativas entre o uso de determinados fármacos e uma maior incidência de ITUs, nem nos casos em que foi necessário o uso de fármacos para indução de imunossupressão, nem naqueles em que houve utilização de inibidores da mTor. Tal como descrito noutros estudos, ${ }^{14}$ relativamente à terapêutica de manutenção, notámos uma incidência superior de doentes que desenvolveram ITUs e ITUs recorrentes no grupo de doentes que se encontrava sob tacrolimus $(57,7 \%$ e $23,1 \%$, respetivamente vs $37,4 \%$ e $14,8 \%$ no grupo que se encontrava sob ciclosporina). Encontra-se documentado que as consequências atribuídas ao uso dos fármacos imunossupressores (nomeadamente, as infeções e as neoplasias) se relacionam mais com a intensidade da imunossupressão do que com os agentes utilizados. ${ }^{4}$ Analogamente, e relativamente à terapêutica de indução, observámos um número superior de ITUs no grupo em que foi utilizada ATG comparativamente aqueles em que foi usado basiliximab, sendo esta tendência concordante com o que é descrito na literatura ${ }^{1,2,4,9}$ uma vez que a terapêutica com ATG, por ser um fármaco depletor, é mais imunossupressora.

Avaliámos ainda o valor de creatininémia mais baixo, usualmente referido como nadir, bem como o valor de creatininémia ao fim do período de seguimento de modo a inferir, ainda que grosseiramente, qual o estado de funcionamento do enxerto renal. Como expectável, verificámos um aumento dos valores de creatinina ao longo do seguimento, com um valor superior no grupo de doentes com ITUs. Apesar de não se apurar uma relação estatisticamente significativa, esta diferença poderá ser justificada pela possível lesão do parênquima renal causada pela ocorrência de infeções. ${ }^{1,2}$ Deste modo, seria expectável, no grupo de doentes com ITUs recorrentes, um valor superior de creatininémia aos cinco anos. No entanto, ambos os grupos apresentam 
valores semelhantes $(0,37 \mathrm{mg} / \mathrm{dl}$ no grupo com ITUs não recorrentes e $0,28 \mathrm{mg} / \mathrm{dl}$ no grupo com ITUs recorrentes).

Apesar de estar documentado um aumento da mortalidade relacionado com a ocorrência de ITUs, ${ }^{1,2,5,6,9,15}$ tal não se verificou no presente estudo. Contabilizámos perda enxerto em $11,0 \%$ da população estudada, o que se mostra consistente com o referido na literatura $(5-17 \%) .{ }^{11}$ Não identificámos nenhuma relação estatisticamente significativa entre a ocorrência de ITUs e a perda de enxerto renal mas observámos que, dos doentes cuja causa de perda do enxerto foi rejeição aguda $(n=5)$, apenas $20,0 \%$ $(n=1)$ registaram ITUs, enquanto nos doentes que perderam enxerto por disfunção crónica $(n=4), 75,0 \%(n=3)$ desenvolveram ITUs ao longo do período de seguimento. Nos casos de perda do enxerto por disfunção crónica a infeção pode, por si só, apresentar-se como um fator potenciador de fenómenos de rejeição dado que ocorrem, durante a resposta ao agente etiológico infecioso, processos de libertação de mediadores inflamatórios, como citocinas e radicais livres que podem lesar o parênquima renal.,2,12

Quanto a outros aspetos clínicos relacionados com as ITUs, verificámos que o primeiro semestre após transplante é aquele com maior ocorrência de ITUs $(25,5 \%)$, o que corresponde ao esperado..$^{1-3,6}$ Contribuem para um maior risco de ITU os fatores peri-cirúrgicos, nomeadamente a instrumentação do trato urinário, bem como níveis de imunossupressão mais elevados, sobretudo nas doses de corticoesteróides. ${ }^{1-3,5}$ Estes dados vêm enfatizar a importância da instituição de uma profilaxia antibiótica desde cedo. ${ }^{1,5} \mathrm{As}$ orientações europeias recomendam a profilaxia para ITUs nos transplantados renais com trimetoprim-sulfametoxazol, 1,2,5,7,9 que para além de prevenir a infeção por Pneumocystis jirovecci, parece contribuir para a diminuição da incidência de ITUs, da duração da doença sintomática, da ocorrência de bacteriemia e necessidade de hospitalização. ${ }^{1,2,4,5} \mathrm{~A}$ duração ideal desta profilaxia não está, contudo, definida. ${ }^{5}$ Apesar de na maioria dos episódios de ITU não ter sido necessário o internamento do doente (correspondendo a $69,4 \%$ dos episódios de ITU não recorrentes e $71,7 \%$ dos episódios de ITU recorrentes), as ITUs foram a principal causa de internamento no Serviço de Nefrologia. Não devemos, contudo assumir que as ITUs nesta população constituem um problema de saúde trivial, uma vez que podem estar associadas a complicações potencialmente letais., 1,6,13 A ausência de mortalidade por ITUs na nossa coorte, mesmo em doentes imunossuprimidos e com manifestações graves, vem corroborar a necessidade de um baixo limiar para internamento nesta população, com instituição de terapêutica num momento precoce da doença.

Do ponto de vista laboratorial, o agente mais frequentemente isolado em urinocultura foi Escherichia coli $(51,6 \%)$, seguido de Klebsiella pneumoniae (15,5\%) e Enterobacter spp. $(9,9 \%)$, o que se revelou semelhante ao referido na literatura. ${ }^{1,2,5,9,16,13}$ Esta hierarquia manteve-se para as ITUs recorrentes. Recentemente, num estudo noutro centro hospitalar nacional Klebsiella pneumoniae foi a espécie responsável pela maioria das ITUs recorrentes. ${ }^{10}$
O facto de não termos encontrado algumas associações já descritas noutros estudos pode ser em parte explicado pela menor dimensão da nossa população, o que impossibilita a análise de diferenças com potência estatística adequada. Por outro lado, os dados são relativos a um único centro em Portugal, o que limita a sua generalização para outros centros, com outros modelos de seguimento. Nesta análise, não foi possível estudarmos algumas variáveis descritas na literatura, uma vez que algumas informações importantes estavam inconsistentemente sinalizadas nos processos clínicos dos doentes, nomeadamente, número de dias de algaliação pós transplante, presença de cateteres duplo $\mathrm{J}$ e necessidade de realização de diálise precoce pós transplante. Não é plausível que se tenha verificado viés de informação sobre os restantes dados recolhidos sendo, contudo, pouco provável a existência de ITUs não reportadas.

Do nosso conhecimento, este é o único estudo realizado em Portugal que permite, sendo um estudo de coorte com um seguimento prolongado, obter informação de resultados clínicos a curto e longo prazo no período pós-transplante relativos à ocorrência de ITUs e ITUs recorrentes.

\section{CONCLUSÃO}

Na nossa população, apenas o género feminino foi identificado como um fator de risco para o desenvolvimento de ITUs, recorrentes ou não. O primeiro semestre após transplante foi aquele com maior ocorrência de ITUs $(25,5 \%)$, sendo Escherichia coli o agente etiológico mais frequente.

O prolongado período de seguimento deste estudo contribuiu com informação relevante e levanta novas questões. Sobressai a necessidade da realização de estudos, de carácter prospetivo, com um maior número de doentes e preferencialmente multicêntricos, que caracterizem meIhor a realidade nacional. Para já, podemos concluir que os resultados da transplantação renal, medidos em termos de sobrevida dos doentes e de duração dos enxertos, têm melhorado progressivamente mas as intercorrências, designadamente as infeciosas, continuam a ser um problema de grande relevo na medicina da transplantação.

\section{AGRADECIMENTOS}

Os autores gostariam de agradecer a colaboração do Secretariado da Consulta da Unidade de Transplantação Renal do Hospital de Santa Cruz e a todos os funcionários da Unidade, que tornaram este estudo exequível.

\section{CONFLITOS DE INTERESSE}

Os autores não declararam a existência de quaisquer conflitos de interesse relativamente ao presente artigo.

\section{FONTES DE FINANCIAMENTO}

Os autores não declararam a existência de quaisquer fontes externas de financiamento que tenham contribuído para a realização deste artigo. 


\section{REFERÊNCIAS}

1. Saemann M, Hori W. Urinary tract infection in renal transplant recipients. Eur J Clin Invest. 2008;38:58-65

2. Souza R, Olsburgh J. Urinary tract infection in the renal transplant patient. Nat Clin Pract Nephrol. 2008;5:252-64.

3. Lorenz E, Gosio F. The impact of urinary tract infections in renal transplant patients. Kidney Int. 2010;78:713-721.

4. Mandell L, Bennett J, Dolin R. Risk factors and approaches to infections in transplant recipients. In: Principles and Practice of Infectious Diseases. $6^{\text {th }}$ ed.New York: Churchill, Livingstone;2004. p.3476-84.

5. Mitra S, Alangaden G. Recurrent urinary tract infections in kidney transplant recipients. Curr Infect Dis Rep Springer Science. 2011;13:579-87.

6. Chuang $P$, Parikh $R$, Langone A. Urinary tract infections after renal transplantation: a retrospective review at two US transplant centers. Clin Transplant. 2005;19:230-5.

7. Grabe M, Bjerklund-Johansen E, Botto H, Çek M, Naber G, Tenke P, et al. Guidelines on urological infections. Eur Urol. 2011;8:56-63.

8. Gabinete de Rim da Sociedade Portuguesa de Transplantação. Lisboa: Registo Português de Transplantação Renal; 2009.

9. Alangaden J, Thyagarajan R, Gruber A, Morawski K, Garnick J, El-Amm $M$, et al. Infectious complications after kidney transplantation: current epidemiology and associate risk factors. Clin Transplant. 2006;20:401-9.

10. Silva C, Afonso N, Macário F, Alves R, Mota A. Recurrent urinary tract infections in kidney transplant recipients. Transplant Proc. 2013:45:10925.

11. Halloran P. Immunosupressive drugs for kidney transplantation. NEJM. 2004;351:2715-29.

12. Pellé G, Vimont S, Levy P, Hertig A, Ouali N, Chassin C, et al. Acute pyelonephritis represents a risk factor impairing long-term kidney graft function. Am J Transplant. 2007;7:899-907.

13. Cosio F, Pesavento T, Osei K, Henry M, Ferguson R. Post-transplant diabetes mellitus: Increasing incidence in renal allograft recipients transplanted in recent years. Kidney Int. 2001;59:732-7.

14. Margereiter R. Efficacy and safety of tacrolimus compared with cyclosporine microemulsion in renal transplantation: a randomized multicenter study. Lancet. 2002;359:741-6.

15. Abbott K, Swanson J, Richter E, Bohen E, Agodoa L, Peters T, et al. Late urinary tract infection after renal transplantation in the United States. Am J Kidney Dis. 2004;44:353-62.

16. Senger S, Arslan H, Azap O, Timurkaynak F, Çagir U, Haberal M. Urinary tract infection in renal transplant recipients. Transplant Proc. 2007;39:1016-7.

17. Fishmann J. Infection in solid organ transplant recipients. NEJM. 2007;357:25

18. Valdez-Ortiz R, Sifuentes-Osornio J, Morales-Buenrostro L, Ayala-Palma H, Dehesa-López E, Alberú J, et al. Risk factors for infections requiring hospitalization in renal transplant recipients: a cohort study. Int J Infect Dis. 2010;15:188-96.

19. Valera B, Gentil M, Cabello V, Cordero E, Cisneros J. Epidemiology of urinary infections in renal transplant recipients. Transplant Proc. 2006;38:2414-5.

20. Kawecki D, Kwiatkowski A, Sawicka-Grzelak A, Durlik M, Paczek L, Chmura A, et al. Urinary tract infections in the early post transplant period after kidney transplantation: etiologic agents and their susceptibility. Transplant Proc. 2011;43:2991-3. 


\title{
Infeções do Trato Urinário numa Coorte de Transplantados Renais
}

\author{
Acta Med Port 2014:27:364-371
}

Publicado pela Acta Médica Portuguesa, a Revista Científica da Ordem dos Médicos

Av. Almirante Gago Coutinho, 151

1749-084 Lisboa, Portugal.

Tel: +351 218428215

E-mail: submissao@actamedicaportuguesa.com

www.actamedicaportuguesa.com

ISSN:0870-399X | e-ISSN: 1646-0758

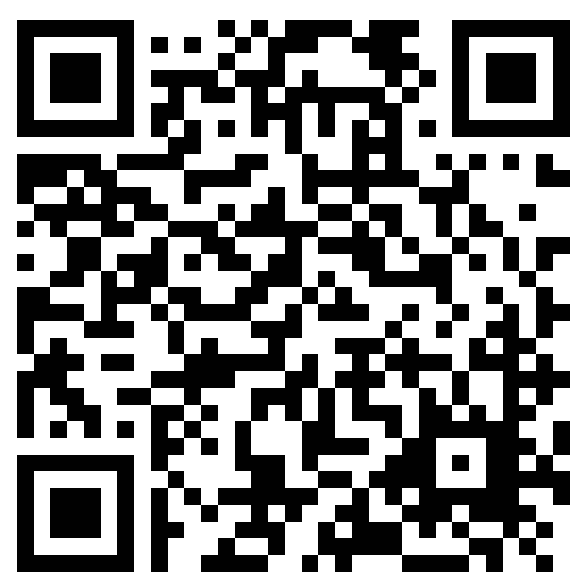

